\title{
Impactos da Ventilação Não Invasiva em Crianças Asmáticas Internadas em Unidades de Terapia Intensiva e Enfermarias: Uma Revisão Integrativa
}

\author{
Juma Apolinária Alves da Silva, ${ }^{1}$ Andreza Izis da Silva Oliveira, ${ }^{2}$ \\ Ery Albuquerque Magalhães Neto²
}

RESUMO

Introdução: A asma é uma das doenças respiratórias crônicas de maior frequência na infância. A fisiopatologia das condições clínicas no estado asmático fornece uma sólida base fisiológica para o uso da ventilação não invasiva (VNI) em crianças. Objetivo: analisar a eficácia e a segurança da VNI em crianças asmáticas internadas em ambiente hospitalar, assim como seus efeitos fisiológicos no sistema respiratório por meio das evidências já existentes. Métodos: Foi realizada pesquisa nas bases de dados SciELO, Pubmed, Lilacs e na Biblioteca Virtual em Saúde (BVS) buscando artigos originais sobre asma e VNI em crianças, em português, inglês e espanhol, publicados entre 2010 e 2020, mediante os descritores padronizados (Descritores em Ciências de Saúde - DeCS): "acute asthma", "bilevel", "pediatrics", "continuous positive airway pressure". Seguiu-se uma metodologia e critérios de inclusão e exclusão, de modo que sete artigos foram selecionados. Destes, apenas um teve nível de evidência A (14,3\%), outro B (14,3\%) e cinco nível C (71,4\%). Resultados: Seis artigos apresentaram desfechos positivos quanto ao uso da VNI nas disfunções respiratórias causadas pela asma. Os pacientes apresentaram melhoras clínica, como otimização das trocas gasosas, menor tempo de estadia no hospital, menor índice de intubação, entre outras. Apenas um estudo mostrou que a VNI é ineficaz e sem benefícios aparentes. Considerações finais: Os estudos indicaram que a VNI é uma boa opção terapêutica, segura e eficaz. As informações atuais, entretanto, encontram-se limitadas, com pouca qualidade e controversas, sem uma padronização do seu manejo, sendo necessária a realização de mais estudos randomizados que não deixem dúvidas quanto à sua utilização nesta população.

Palavras-chave: Ventilação não invasiva. Asma. Criança.

IMPACTS OF NON-INVASIVE VENTILATION ON ASTHMATIC CHILDREN ADMITTED TO INTENSIVE CARE UNITS AND WARDS: NA INTEGRATIVE REVIEW VENTILAÇÃO NÃO INVASIVA EM CRIANÇAS ASMÁTICAS

\section{ABSTRACT}

Introduction: Asthma is one of the most common chronic respiratory diseases in childhood. The pathophysiology of clinical conditions in asthmatic status provides a solid physiological basis for the use of noninvasive ventilation (NIV) in children. Objective: to analyze the effectiveness and safety of NIV in asthmatic children hospitalized in a hospital environment, as well as its physiological effects on the respiratory system, through the existing evidence. Methods: Research was carried out in the SciELO, Pubmed, Lilacs and Virtual Health Library (VHL) databases, for original articles on asthma and NIV in children. In Portuguese, English and Spanish, published between 2010 and 2020. Through the standard DeCS descriptors: "acute asthma", "bilevel", "pediatrics", "continuous positive airway pressure". Following a methodology and inclusion and exclusion criteria, so that seven articles were selected. Of these, only one presented evidence level A (14.3\%), another B (14.3\%) and five level C (71.4\%). Results: Six articles showed positive outcomes regarding the use of NIV in respiratory disorders caused by asthma. The patients showed clinical improvements such as, gas exchange optimization; shorter hospital stay, lower intubation rate, among others. Only one study indicated that NIV is ineffective and has no apparent benefits. Final considerations: Studies have indicated that NIV is a good, safe and effective therapeutic option. However, current information is limited, of low quality and controversial, without a standardization of its management. It is necessary to conduct more randomized studies that leave no doubt as to its use in this population.

Keywords: Non-Invasive. Asthma. Children.

RECEBIDO EM: 3/6/2020

MODIFICAÇÕES SOLICITADAS EM: 10/11/2020

ACEITO EM: 5/1/2021

\footnotetext{
${ }_{1}^{1}$ Autora correspondente. Faculdade de Comunicação, Tecnologia e Turismo de Olinda - Facottur. Av. Pres. Getúlio Vargas, 1360 - Bairro Novo. Olinda/PE, Brasil. CEP 53030-010. http://lattes.cnpq.br/8530526208763582. http://orcid.org/00000001-6761-2966. jumaalves1990dl@gmail.com

2 Faculdade de Comunicação, Tecnologia e Turismo de Olinda - Facottur. Olinda/PE, Brasil.
} 


\section{INTRODUÇÃO}

A asma é uma das doenças respiratórias crônicas de maior frequência na infância, sendo classificada como um processo inflamatório sistêmico crônico, caracterizado por uma hiper-responsividade das vias aéreas inferiores e por redução variável ao fluxo aéreo. A obstrução ao fluxo aéreo resulta da hiper-reatividade, inflamação e edema da mucosa brônquica, espasmos dos músculos das vias aéreas e aumento e acúmulo de secreções nestas vias (DAVID et al., 2018; RODRIGUEZ; PEZO; PAMELA, 2018).

A enfermidade é representada como a terceira causa de internações por doenças clínicas e a quarta causa de morte por doenças respiratórias. A previsão para 2025 é o acréscimo de mais de 100 milhões nesse cálculo. Estima-se que $14 \%$ das crianças no mundo padecem dos sintomas dessa enfermidade. No Brasil, a doença tem prevalência entre escolares de seis e sete anos e adolescentes de 13 e 14 anos, de 24,3\% e 19,0\%, respectivamente (PITCHON et al., 2018).

O tratamento convencional para a asma depende da gravidade da condição. Em casos de exacerbações asmáticas, geralmente dá-se início ao tratamento por meio de fármacos de primeira linha, que, geralmente, compõem-se de broncodilatadores e corticosteroides, seguidos pelos agentes de segunda linha, como o brometo de ipratrópio e sulfato de magnésio. Existem alguns pacientes, entretanto, que não respondem ao tratamento farmacológico de primeira linha, tampouco os de segunda linha. Este pacientes podem, na falha do tratamento medicamentoso, ser induzidos a um tratamento invasivo por meio da intubação orotraqueal (OLIVEIRA et al., 2020).

A ventilação não invasiva (VNI) é um recurso terapêutico não farmacológico e não invasivo, que pode ser utilizado na tentativa de amenizar as complicações e sintomas causados pela asma.

A ventilação não invasiva (VNI) consiste em uma modalidade de suporte ventilatório que, por meio da utilização de interfaces não invasivas, permite a ventilação do doente, sem necessidade de recorrer à ventilação invasiva por intermédio da intubação orotraqueal (MAGALHÃES; SOARES, 2018). Há duas maneiras de se ofertar este suporte ventilatório: por pressão positiva contínua nas vias aéreas (CPAP) ou por dois níveis de pressão (Bipap) (FARIAS; CALLES, 2018). Durante exacerbação asmática, uma pressão expiratória positiva é criada no final da expiração intrínseca. O uso da VNI neutraliza essa PEEP intrínseca, criando uma PEEP extrínseca.

A VNI, no modo CPAP, fornece uma pressão positiva constante nas vias aéreas durante todo o ciclo respiratório, de modo que as pressões intraluminais mais altas impedem o colapso das vias aéreas superiores, promovendo um maior recrutamento alveolar e, consequentemente, o aumento da capacidade residual funcional (CRF), neutralizando, assim, o desenvolvimento de atelectasias. Por esse mecanismo, o CPAP consegue melhorar a oxigenação e a hipoxemia apresentadas pelo paciente (PARGA et al., 2017).

Quando ofertada no modo Bipap ou bilevel, ocorre a oferta de dois níveis pressóricos, fazendo com que o paciente tenha volumes pressóricos variáveis 
tanto na inspiração quanto na expiração. Por este mecanismo o Bipap é capaz de aumentar o CRF, melhorando a oxigenação e a hipoxemia do paciente, assim como também aumentar o volume corrente e, consequentemente, o volume/ minuto, sendo eficaz também na correção da hipercapnia (DAVID et al., 2018; FERREIRA; COLTO; YKEDA, 2013).

Os fármacos são inalados à base da terapia para muitas doenças pulmonares em crianças. Existem poucas pesquisas sobre o uso de fármacos aerossóis durante a VNI pediátrica. Os resultados parecem depender de inúmeros fatores associados, como o tipo de interface e modo ventilatório utilizado, a posição da conexão para a aerossolterapia no circuito, boa vedação da máscara, entre outros (BERLINSKI, 2017).

Embora o potencial de evitar a ventilação invasiva seja um benefício real dessa abordagem, a VNI na pediatria geralmente é desafiadora em razão da necessidade de uma variedade de interfaces de tamanho adequado e da intolerância de algumas crianças ao dispositivo. Alguns pacientes podem demonstrar desconforto pela pressão e pelo fluxo de ar. Em alguns casos pode haver a necessidade do uso de algum tipo de sedação (REHDER, 2017).

Este estudo consiste em analisar a eficácia e a segurança da ventilação mecânica não invasiva em pacientes pediátricos asmáticos internados em ambiente hospitalar, assim como seus efeitos fisiológicos no sistema respiratório, por meio das evidências já existentes.

\section{MATERIAIS E MÉTODO}

O presente estudo trata-se de uma revisão de literatura integrativada. Foram realizadas buscas por literaturas disponíveis em bancos de dados na internet, como Pubmed, Lilacs, Scielo e na Biblioteca Virtual em Saúde (BVS), por artigos científicos originais que abordassem a asma e o uso da ventilação não invasiva em crianças asmáticas (até 18 anos). Os artigos foram obtidos por meio dos descritores padronizados nos Descritores em Ciências de Saúde (DeCS): “ acute Asthma", "status asthmaticus", "continuous positive airway pressure", "insufficiency respiratory ", "infant", "bilevel", além das palavras-chave: ventilação não invasiva, asma e criança. As combinações entre as palavras foram realizadas nas bases supracitadas, utilizando os operadores "Or" e "And".

A busca limitou-se a artigos escritos em português, inglês e espanhol, com período de publicação de 2010 a 2020. Posteriormente foram selecionados quanto aos critérios de inclusão e exclusão. Para a seleção dos artigos foram estabelecidos os seguintes critérios de inclusão: apresentar informações sobre a utilização da ventilação não invasiva e seus efeitos no sistema respiratório apenas na população pediátrica asmática em unidade de terapia intensiva ou enfermaria. Os critérios de exclusão foram: artigos que apresentassem, em sua amostra, adultos ou ano de publicação inferior ao ano de 2010, sendo estes excluídos.

Para a avaliação da qualidade metodológica dos artigos encontrados decidiu-se pela aplicação do sistema Grading of Recommendations Assessment, Development and Evaluation (Grade) de qualidade da evidência científica (GUYATT et al., 2008). 


\section{RESULTADOS}

Na busca foram inicialmente identificados 115 artigos para leitura exploratória dos resumos, e então selecionados 27 que foram lidos integralmente. Depois da leitura destes, apenas sete artigos preenchiam os criterios de inclusão e exclusão e foram selecionados.Um dos artigos apresentou nível de evidência $\mathrm{A}$ (14,3\%), outro nível B (14,3\%) e cinco apresentaram nível de evidência C (71,4\%).

As características de cada estudo podem ser encontradas na Tabela 1.

Tabela 1 - Características de cada estudo

\begin{tabular}{|c|c|c|c|c|c|}
\hline Autor & Delineamento & Amostra & Intervenção & resultados & conclusão \\
\hline $\begin{array}{l}\text { Kang et al., } \\
2019\end{array}$ & $\begin{array}{l}\text { Estudo } \\
\text { descritivo } \\
\text { retrospectivo }\end{array}$ & $\begin{array}{l}33 \text { crianças } \\
\text { (idade } \leq \\
18 \text { anos) } \\
\text { admitidas } \\
\text { em Utip, por } \\
\text { ataque de } \\
\text { asma. }\end{array}$ & $\begin{array}{l}\text { Sem interação, } \\
\text { revisão de } \\
\text { prontuários. } \\
\text { Bipap por } \\
\text { máscara nasal. } \\
\text { Agrupadas de } \\
\text { acordo com o } \\
\text { uso do Bipap } \\
\text { (SIM/NÃO). } 24 \\
\text { com Bipap e } 21 \\
\text { sem Bipap. }\end{array}$ & $\begin{array}{l}\text { Houve melhora } \\
\text { significativa dos } \\
\text { níveis de } \mathrm{PCO}_{2} \mathrm{e} \\
\text { na FR do grupo } \\
\text { Bipap após o } \\
\text { início do suporte } \\
\text { ventilatório } \\
\text { em relação ao } \\
\text { não Bipap. Os } \\
\text { níveis de } \mathrm{SpO}_{2} \\
\text { melhoraram } \\
\text { significativamen } \\
\text { te nos dois } \\
\text { grupos. }\end{array}$ & $\begin{array}{l}\text { Segura e eficiente } \\
\text { em melhorar a FR } \\
\text { e a oxigenação. }\end{array}$ \\
\hline $\begin{array}{l}\text { Pilar et al., } \\
2017\end{array}$ & $\begin{array}{l}\text { Estudo } \\
\text { observacional } \\
\text { de uma coorte } \\
\text { retrospectiva }\end{array}$ & $\begin{array}{l}42 \text { crianças } \\
\text { com } \\
\text { exacerbação } \\
\text { da asma, em } \\
\text { Utip. }\end{array}$ & $\begin{array}{l}20(47,6 \%) \\
\text { receberam } \\
\text { HFNC e } 22 \\
(52,3 \%) \text { VNI } \\
\text { como suporte } \\
\text { respiratório } \\
\text { inicial. }\end{array}$ & $\begin{array}{l}\text { Não houve falhas } \\
\text { no tratamento } \\
\text { no grupo VNI. } 8 \\
\text { crianças (40\%) no } \\
\text { grupo HFNC, no } \\
\text { entanto, exigiram } \\
\text { escalação para } \\
\text { a VNI. O tempo } \\
\text { na Utip foi } \\
\text { semelhante } \\
\text { entre os dois. O } \\
\text { grupo que falhou, } \\
\text { entretanto, na } \\
\text { HFNC, o tempo de } \\
\text { estadia e suporte } \\
\text { foi } 3 \text { vezes maior. }\end{array}$ & $\begin{array}{l}\text { Embora estudos } \\
\text { observacionais } \\
\text { tenham suas } \\
\text { limitações, este } \\
\text { resultado pode } \\
\text { indicar que a VNI } \\
\text { apresenta maiores } \\
\text { taxas de sucesso, e } \\
\text { que a HFNC pode } \\
\text { colaborar para o } \\
\text { atraso do uso da } \\
\text { VNI e maior tempo } \\
\text { de estadia no } \\
\text { hospital. }\end{array}$ \\
\hline $\begin{array}{l}\text { Abramo et } \\
\text { al., } 2017\end{array}$ & $\begin{array}{l}\text { Análise } \\
\text { descritiva }\end{array}$ & $\begin{array}{l}1.157 \\
\text { crianças } \\
\text { asmáticas }\end{array}$ & $\begin{array}{l}\text { Sendo } \\
\text { distribuídas } 325 \\
\text { em Utip e } 832 \\
\text { em enfermaria, } \\
\text { que fizeram uso } \\
\text { do Bipap entre } \\
2005 \text { e } 2013 .\end{array}$ & $\begin{array}{l}\text { Boa adessão, } \downarrow \\
\text { FR, } \downarrow \text { do escore } \\
\text { de gravidade da } \\
\text { asma, } \uparrow \text { volume } \\
\text { corrente. Apenas } \\
6 \text { pacientes foram } \\
\text { intubados. }\end{array}$ & $\begin{array}{l}\text { Segura, eficaz, } \\
\text { no tratamento } \\
\text { precoce da } \\
\text { asma. Com boa } \\
\text { adesão, menor } \\
\text { tempo de terapia, } \\
\text { baixo índice } \\
\text { de intubação e } \\
\text { complicações. }\end{array}$ \\
\hline
\end{tabular}




\begin{tabular}{|c|c|c|c|c|c|}
\hline $\begin{array}{l}\text { Golden et } \\
\text { al., } 2015\end{array}$ & $\begin{array}{l}\text { Estudo } \\
\text { observacional } \\
\text { prospectivo }\end{array}$ & $\begin{array}{l}933 \text { crianças } \\
\text { admitidas } \\
\text { na Utip com } \\
\text { exacerbações } \\
\text { agudas de } \\
\text { asma. }\end{array}$ & $\begin{array}{l}\text { Divididos em } \\
2 \text { grupos de } \\
\text { tratamento com } \\
\text { Bipap (SIM=45) } \\
\text { e gravidade } \\
\text { moderada. } \\
\text { E sem Bipap } \\
\text { (NÃO= 888) com } \\
\text { gravidade leve a } \\
\text { moderada entre } \\
\text { os participantes } \\
\text { que não } \\
\text { apresentavam } \\
\text { sinais de } \\
\text { insuficiência } \\
\text { respiratória. } \\
\text { O modelo } \\
\text { de escore de } \\
\text { propensão } \\
\text { produziu uma } \\
\text { estatística } \\
\text { C de } 0,895, \\
\text { e os grupos } \\
\text { pareados foram } \\
\text { muito bem } \\
\text { equilibrados nas } \\
\text { covariáveis. }\end{array}$ & $\begin{array}{l}\text { Os participantes } \\
\text { tratados } \\
\text { com Bipap } \\
\text { apresentaram } \\
\text { maior } \\
\text { probabilidade de } \\
\text { serem internados } \\
\text { no hospital ou } \\
\text { na Utip, e com } \\
\text { nenhum benefício } \\
\text { aparente quanto } \\
\text { à duração do } \\
\text { tempo de estadia } \\
\text { hospitalar. }\end{array}$ & $\begin{array}{l}\text { Os autores } \\
\text { concluem, porém, } \\
\text { que o estudo é } \\
\text { limitado, pois não } \\
\text { podem resolver se } \\
\text { esses achados são } \\
\text { consequência do } \\
\text { momento clínico } \\
\text { após o uso da VNI } \\
\text { ou do aumento da } \\
\text { retenção de ar e da } \\
\text { incompatibili dade } \\
\text { da ventilação- } \\
\text { perfusão, } \\
\text { desordens que } \\
\text { agravam uma } \\
\text { exacerbação. }\end{array}$ \\
\hline $\begin{array}{l}\text { Williams et } \\
\text { al., } 2011\end{array}$ & $\begin{array}{l}\text { Estudo } \\
\text { descritivo } \\
\text { retrospectivo e } \\
\text { prospectivo }\end{array}$ & $\begin{array}{l}165 \text { crianças } \\
\text { com } \\
\text { exacerbações } \\
\text { moderadas } \\
\text { e graves de } \\
\text { asma. Com } \\
20 \text { kg ou } \\
\text { menos e que } \\
\text { receberam } \\
\text { tratamento } \\
\text { Bipap. }\end{array}$ & $\begin{array}{l}\text { Descrição } \\
\text { de dados } \\
\text { retrospectivos } \\
\text { de } 112 \\
\text { pacientes e } \\
\text { prospectivos } \\
\text { de } 53 \text {. }\end{array}$ & $\begin{array}{l}\text { Sem mortes e } \\
\text { complicações } \\
\text { como } \\
\text { pneumotórax } \\
\text { ou pneumonia, } \\
\text { porém um } \\
\text { episódio de } \\
\text { aspiração; } 4 \text { IOT } \\
\text { mesmo após VNI; } \\
6 \text { excluídos por } \\
\text { tempo de VNI } \\
\text { menor que } 30 \\
\text { minutos. Todos } \\
\text { melhoram no } \\
\text { escore da asma. }\end{array}$ & $\begin{array}{l}\text { Sendo considerada } \\
\text { segura e podendo } \\
\text { melhorar o } \\
\text { desfecho clínico } \\
\text { desses pacientes. }\end{array}$ \\
\hline $\begin{array}{l}\text { Basnet et al., } \\
2012\end{array}$ & $\begin{array}{l}\text { Estudo clínico } \\
\text { prospectivo } \\
\text { randomizado, } \\
\text { controlado. }\end{array}$ & $\begin{array}{l}20 \text { crianças, } \\
\text { admitidas } \\
\text { na Utip } \\
\text { com estado } \\
\text { asmático. }\end{array}$ & $\begin{array}{l}\text { As crianças } \\
\text { foram } \\
\text { randomizadas } \\
\text { para receber } \\
\text { VNI + terapia } \\
\text { medicamentosa } \\
\text { padrão versus } \\
\text { grupo só terapia } \\
\text { padrão. }\end{array}$ & $\begin{array}{l}\downarrow \text { da FR, melhora } \\
\text { do escore clínico } \\
\text { da asma, sem } \\
\text { efeitos adversos } \\
\text { importantes. } 9 \text { em } \\
\text { cada } 10 \text { pacientes } \\
\text { toleraram a VNI. }\end{array}$ & $\begin{array}{l}\text { O uso precoce } \\
\text { de VNI + } \\
\text { broncodilatadores } \\
\text { de ação rápida } \\
\text { e esteroides } \\
\text { sistêmicos, pode } \\
\text { ser seguro, efetivo } \\
\text { e bem tolerado. }\end{array}$ \\
\hline
\end{tabular}




\begin{tabular}{|c|c|c|c|c|c|}
\hline $\begin{array}{l}\text { Mayordomo- } \\
\text { Colunga et } \\
\text { al., } 2011 .\end{array}$ & $\begin{array}{l}\text { Estudo } \\
\text { observacional } \\
\text { prospectivo. }\end{array}$ & $\begin{array}{l}72 \text { crianças } \\
\text { asmáticas na } \\
\text { Utip, que não } \\
\text { responderam } \\
\text { à terapia } \\
\text { convencional } \\
\text { com trabalho } \\
\text { respiratório. }\end{array}$ & $\begin{array}{l}\text { Durante a } \\
\text { terapia com VNI, } \\
\text { o salbutamol } \\
\text { foi nebulizado } \\
\text { continuamente } \\
\text { e o brometo } \\
\text { de ipratrópio } \\
\text { a cada } 2 \\
\text { horas e metil- } \\
\text { prednisolona. }\end{array}$ & $\begin{array}{l}\text { Na primeira hora } \\
\text { de uso houve: } \downarrow \\
\text { no escore clínico } \\
\text { da asma, } \downarrow \text { na FC } \\
\text { e na FR. }\end{array}$ & $\begin{array}{l}\text { VNI é uma terapia } \\
\text { viável e eficaz } \\
\text { em crianças } \\
\text { com asma que } \\
\text { não respondem } \\
\text { ao tratamento } \\
\text { convencional. }\end{array}$ \\
\hline
\end{tabular}

VNI - ventilação não invasiva; Utip - unidade de terapia intensiva pediátrica; FR - frequência respiratória; Bipap - Bilevel Positive Airway Pressure; FC - frequência cardíaca; PaCO _ pressão parcial de gás carbônico; $\mathrm{CO}_{2}$ - gás carbônico; $\mathrm{FIO}_{2}$ - fração inspirada de oxigênio; $\downarrow$ redução; $\uparrow$ aumento.

\section{DISCUSSÃO}

Apesar de o fato da prevalência de asma em crianças ter aumentado mundialmente nas últimas décadas, as indicações para a VNI na asma aguda apresentam fragilidades. Observou-se que não há estudo em larga escala para validar os seus efeitos no tratamento da asma nessa população, nem protocolo padronizado a ser sugerido nas diretrizes atuais (KORANG et al., 2016).

Os estudos demonstram, entretanto, que, após o uso da VNI, ocorrem meIhoras significativas no escore clínico da asma, redução da frequência cardíaca (FC) e da FR, além de redução dos níveis séricos de $\mathrm{PCO}_{2}$ e aumento nos níveis de $\mathrm{SpO}_{2}$, com consequente diminuição na necessidade de oxigênio (ABRAMO et al., 2017; MAYORDOMO-COLUNGA et al., 2011).

Em um estudo recente, Kang et al. (2019) observaram que, logo após o início da VNI no modo Bibap, houve redução significativa dos níveis séricos de $\mathrm{PCO}_{2}$ e melhora dos níveis de $\mathrm{SpO}_{2}$ nas crianças asmáticas que fizeram uso da VNI, melhorando, assim, a frequência respiratória e a oxigenação, demonstrando ser eficaz na melhora da saturação e na prevenção de maior retenção de $\mathrm{CO}_{2}$ nestes pacientes.

O suporte ventilatório não invasivo pode ser ofertado por meio da ventilação não invasiva (VNI) e pela cânula nasal de alto fluxo (HFNC), sendo cada vez mais utilizado em muitas formas de insuficiência respiratória, incluindo asma aguda grave. A HFNC, entretanto, não pode fornecer agentes agonistas $\beta-2$ aerossolizados em asmáticos moderados a graves. Em conformidade, a VNI, no modo CPAP ou Bipap, tem demonstrado ser benéfica nesses tipos de asmáticos (ABRAMO et al., 2017). Alguns estudos demonstram que a VNI, ofertada por pressão positiva em dois níveis nas vias aéreas (Bipap) em asmáticos pediátricos agudos, pode ser um tratamento eficaz.

Pilar et al. (2017), ao fazer o comparativo do uso da VNI versus a HFNC em uma amostra de 42 crianças, divididas em dois grupos, observaram que não houve falha do tratamento no grupo VNI. Oito crianças do grupo HFNC, entretanto, tiveram falha no tratamento, além de maior tempo de permanência na Utip, progredindo para o uso da VNI. 
O emprego da VNI vem sendo cada vez mais comum dadas as inúmeras vantagens quando comparada à ventilação invasiva. A mesma está associada a baixos índices de intubação; diminuição no tempo de internamento hospitalar e de adesão a unidades de terapia intensiva pediátricas, além de redução no número de complicações e de mortes.

Algumas dessas vantagens podem ser observadas no estudo de Abramo et al. (2017), que, após realizarem estudo com amostra de 1.157 crianças asmáticas que fizeram uso da VNI no modo Bipap, observaram excelentes resultados na redução do tempo de internamento hospitalar e de adesão dessas crianças à unidade de terapia intensiva pediátrica, além de boa aceitação dos pacientes ao recurso terapêutico, com um índice de complicações muito baixo quando comparado ao número total da amostra. Apenas seis pacientes que fizeram uso do Bibap foram intubados.

Em oposição, Golden et al. (2015) observaram, em seu estudo, que as crianças que fizeram uso do Bipap estavam mais propensas a internamento hospitalar e de UTI, além de não observarem nenhum benefício aparente com o seu uso e nem na redução do tempo de estadia hospitalar. Trata-se, entretanto, de um estudo muito limitado.

Williams et al. (2011), ao investigarem os achados clínicos e de segurança da VNI no modo Bipap em 165 crianças asmáticas com exacerbação de asma, relataram que não houve ocorrências de morte e complicações, como pneumotórax ou pneumonia, com apenas um episódio de aspiração e quatro intubações orotraqueal. Os autores concluíram, porém, que o Bipap é seguro, eficaz e meIhora os desfechos clínicos nesses pacientes.

Outro benefício associado a VNI é que a mesma pode ser utilizada em conjunto com a terapia medicamentosa convencional, visando a melhorar a obstrução ao fluxo aéreo e reduzir o esforço respiratório. Cogita-se que a VNI possa potencializar a deposição de fármacos aerossóis (broncodilatador) nas vias aéreas por terapia inalatória durante a exacerbação da asma (BERLINSKI, 2017).

Basnet et al. (2012), ao realizar um pequeno estudo randomizado comparando o uso da VNI em conjunto com a terapia medicamentosa versus a terapia medicamentosa isoladamente, concluíram que a utilização das duas terapias em conjunto apresenta bons resultados, com uma rápida e significativa melhora no escore clínico da asma e diminuição na FR e na FC, demonstrando que o uso das duas terapias juntas oferece uma resposta mais rápida e eficaz na resolução da exacerbação asmática.

O status asthmaticus é um ataque de asma agudo e intratável, refratário à intervenção padrão que pode levar à insuficiência respiratória progressiva. O início precoce da VNI, em conjunto com o tratamento padrão de primeira linha na crise asmática refratária, pode ser eficaz em sua resolução (PARGA et al., 2017).

No estudo de Mayordomo-Colunga et al. (2011), a VNI foi administrada em conjunto com a terapia convencional em crianças com exacerbação da asma que não haviam respondido à terapia convencional isoladamente e com acentuado trabalho respiratório. Na primeira hora de administração houve melhora no escore de asma e redução da FC e da FR, demonstrando que a VNI é uma 
terapia viável em crianças com exacerbação de asma que não responde ao tratamento convencional.

\section{CONSIDERAÇÕES FINAIS}

Nesta revisão de literatura, dos sete resultados que emergiram seis deles indicam que a ventilação não invasiva é uma opção terapêutica segura e eficaz que promove melhoras fisiológicas significativas, como a redução dos níveis séricos de $\mathrm{PCO}_{2}$ e aumento dos níveis séricos de $\mathrm{SpO}_{2}$, além de redução na frequência cardíaca e respiratória, melhorando, assim, a oxigenação e a função pulmonar, principalmente na porcentagem de pacientes que não responde à terapia medicamentosa de primeira escolha isoladamente e quando iniciada precocemente. Apenas um estudo indica que a ventilação não invasiva é ineficaz e sem benefícios aparentes. As informações atuais, entretanto, encontram-se limitadas, com pouca qualidade e controversas, sem uma padronização do seu manejo, sendo necessária a realização de mais estudos randomizados que não deixem dúvidas quanto à sua utilização para o tratamento da asma nesta população.

\section{REFERÊNCIAS}

ABRAMO, T. et al. Programa pediátrico de melhoria contínua da qualidade do ED BIPAP com análise do paciente: 2005-2013. BMJ Open, v. 7, 2017.

BASNET, S. et al. Segurança eficácia e tolerabilidade do início precoce da ventilação não invasiva por pressão positiva em pacientes pediátricos admitidos com status asmático: um estudo piloto. Pediatr Crit Care Med., v. 13, n. 4, p. 393-398, 2012.

BERLINSKI, A. Terapia pediátrica em aerossol. Rev. Respiratory Care, v. 62, n. 6, p. 662677, jun. 2017.

DAVID, M. M. C. et al. A ventilação não invasiva e a fisioterapia respiratória reduzem o broncoespasmo induzido pelo exercício e a inflamação pulmonar em crianças com asma: ensaio clínico randomizado. Avanços Terapêuticos na Doença Respiratória, v. 12, jan./dez. 2018.

FARIAS, D. H.; CALLES, A. C. N. Influência da ventilação mecânica não invasiva (VMNI) no pós-operatório de pacientes submetidos a cirurgia cardíaca: uma revisão integrativa. Rev. Ciências Biológicas e de Saúde Unit., Alagoas, v. 4, n. 2, p. 87-100, 2018.

FERREIRA, L. G. F.; COUTO, A. S.; YKEDA, D. S. Efeitos da ventilação mecânica não invasiva no pós-operatório de cirurgia cardíaca: revisão da literatura. Fisioter S. Fun., v. 2, n. 2, p. 44-50, jul./dez. 2013.

GOLDEN, C. et al. Resultados clínicos após tratamento com pressão positiva em dois níveis nas vias aéreas para exacerbações agudas da asma. Jama Pediatr., v. 169, n. 2, p. 186-188, 2015.

GUYATT, G. H. et al. GRADE Working Group. Going from evidence to recommendations. BMJ, v. 336, n. 7.652, p. 1.049-1.051, 2008.

KANG, C. M et al. A ventilação com pressão positiva nas vias aéreas em dois níveis melhora eficientemente o desconforto respiratório nas primeiras horas do tratamento de crianças com exacerbação grave da asma. J Formos Med. Assoc., v. 119, n. 9, p. 1.415-1.421, 2019. KORANG, S. K et al. Ventilação com pressão positiva não invasiva para asma aguda em crianças. Banco de Dados Cochrane de Revisões Sistemáticas, edição 1, 2016.

MAGALHÃES, F. L. S.; SOARES, D. J. Os benefícios da ventilação não invasiva nos pacientes internados na UTI e em ambulatórios. 2018. 14 f. Trabalho de Conclusão de Curso (Especialização em Saúde da Família) - Instituto de Ciências da Saúde, Universidade da Integração Internacional da Lusofonia Afro-Brasileira, São Francisco do Conde, 2018. 
MAYORDOMO-COLUNGA, J. et al.. Non-invasive ventilation in pediatric status asthmaticus: a prospective observational study. Pediatr. Pulmonol., v. 49, n. 10, p. 949-55, 2011. OLIVEIRA, J. M. et al. Ventilação não invasiva no paciente asmático agudizado em unidade de terapia intensiva. Braz. J. of Develop., v. 6, n. 4, p. 21.408-21.419, Curitiba, abr. 2020. PARGA, D. et al. Ventilação mecânica não invasiva no tratamento do estado asmático em pediatria. Arch Pediatr Urug., v. 88, n. 5, out. 2017.

PILAR, J. et al. Terapia de cânula nasal de alto fluxo versus ventilação não invasiva em crianças com exacerbação aguda de asma aguda: um estudo de coorte observacional. Rev. Medicina Intensiva, v. 47, edição 7, p. 418-424, out. 2017.

PITCHON, R. R. et al. Mortalidade por asma em crianças e adolescentes: uma causa de morte quase sempre evitável. Rev. Med. Minas Gerais, 2018.

REHDER, K. J. Terapias adjuntas para Estado Refratário Asthmaticus em crianças. Respiratory Care, v. 62, n. 6, p. 849-865, jun. 2017.

RODRIGUEZ, R.; PEZO, M. R.; PAMELA, M. Eficacia de la ventilación mecánica con presión positiva no invasiva in pacientes con asma agudo que acuden al servicio de emergencia. Lima, Peru: Universidad Norbert Wiener, 2018.

SHEIN, S. L. et al. Tratamento atual de crianças com asma crítica e quase fatal. Rev. Bras. Ter. Intensiva, v. 28, n. 2, p. 167-178, 2016.

WILLIAMS, A. M. et al. Safety and clinical findings of BIPAP utilization in children $20 \mathrm{~kg}$ or less for asthma axacerbations. Intensive Care Med., v. 37, n. 8, p. 1.338-1.343, 2011. 